\title{
Intelligent Backstepping Control for Genesio-Tesi Chaotic System using a Chaotic Particle Swarm OptimizationAlgorithm
}

\author{
Reza Gholipour, AlirezaKhosravi,HamedMojallali
}

\begin{abstract}
In this paper, an intelligent backstepping controller, tuned using a chaotic particle swarm optimization (CPSO), is proposed to control of chaos in Genesio-Tesi chaotic system. Thebackstepping method consists of parameters with positive values. The parameters are usually chosen optional by trial and error method. The improper selection of the parameters leads to inappropriate responses or even may lead to instability of the system. The proposed intelligent backstepping controller without trial and error determines the parameters of backstepping controller automatically and intelligently by minimizing the Integral of Time multiplied Absolute Error (ITAE) and squared controller output. Finally, the efficiency of the proposed intelligent backstepping controller (IBSC) is illustrated by controlling the chaos of Genesio-Tesi chaotic system.
\end{abstract}

Index Terms-Backstepping Method, Chaotic Particle Swarm Optimization, Genesio-TesiSystem, Chaos Control.

\section{INTRODUCTION}

Chaotic behavior is a very interesting nonlinear phenomenon which has been intensively studied during the last two decades. The effect of chaotic system is usually undesirable in practice due to its sensitivity to initial conditions, unpredictable behavior and thereby restricts the operation of physical plants. Because of the difficulty of accurate prediction of a chaotic system behavior, chaos may cause system instability or degradation in performance, and it should be eliminated in many cases.

Many approaches and techniques have been proposed for the control of chaos such as OGY method [1], bang-bang control [2], optimal control [3], intelligent control based on neural network [4], feedback linearization [5], differential geometric method [6], adaptive control [7]-[10], $H_{\infty}$ control method [11], and many others [12], [13].

The backstepping approach is one of the most popular nonlinear techniques of controller design. It is capable of generating a globally asymptotically stabilizing control laws to suppress and synchronize chaotic system [14]-[17]. The idea of backstepping design is to select recursively some appropriate functions of state variables as pseudo-control inputs for lower dimension subsystems of the overall system. Each backstepping stage results in a new pseudo-controller

Manuscript received August 22, 2012; revised September 27, 2012.

Reza Gholipourand Alireza Khosravi are with the Depatment of Electrical and Computer Engineering, Babol (Noushirvani) University of Technology, Babol, Iran (e-mails: reza_gh_08@yahoo.com; akhosravi@nit.ac.ir).

Hamed Mojallali is with the Electrical Engineering Department, Faculty of Engineering, University of Guilan, Rasht, Iran (e-mail: mojallali@guilan.ac.ir). design, expressed in terms of the pseudo-controller from preceding design stages. When the procedure is terminated, a feedback controller is designed which achieves the original design objective by virtue of a final Lyapunov function formed by summing up the Lyapunov functions associated with each individual design stage.

PSO is a kind of evolutionary algorithm based on a population of potential solutions and motivated by the simulation of social behavior instead of the survival of the fittest individual. It is a population-based evolutionary algorithm. Similar to the other population-based evolutionary algorithms, PSO is initialized with a population of random solutions. Unlike the most of the evolutionary algorithms, each potential solution (individual) in PSO is also associated with a randomized velocity, and the potential solutions, called particles, are then "flown" through the problem space [18]. The performance of the traditional PSO greatly depends on its parameters, and it often suffers the problem of being trapped in local optima [19], [20]. In order to avoid these disadvantages, the chaotic particle swarm optimization (CPSO) method based on the logistic equation has been proposed [20], [21]. Such an algorithm which is known as Chaotic Particle Swarm Optimization (CPSO) is used in this paper in order to determine the intelligent backstepping controller parameters. This algorithm has been utilized widely in recent researches[22-27].

The backsteppingcontroller consists of positive parameters which are determined optional and by trial and error. The system response depends on the choice of these parameters. Improper choice of the parameters causes improper performance and sometimes instability of the system. In this paper, the CPSO algorithm is utilized for determination of proper values of the parameters. In fact, this algorithm determines the parameters of backstepping controller by minimizing the objective function. The objective function, used for controller tuning has been taken as a weighted sum of the Integral of Time multiplied Absolute Error (ITAE) and squared control signal.

The rest of the paper is organized as follows. Section 2 describes the backstepping method. PSO and CPSO are described in Section 3. The proposed intelligent backstepping controller is described in Section 4. In Section 5,simulation results are provided to validate the effectiveness of the proposed method. The paper ends with the conclusion as Section 6 followed by the references.

\section{BACKSTEPPING METHOD}

Consider the following n-order system with strict-feedback form: 


$$
\begin{aligned}
& \dot{x}_{1}=f_{1}\left(x_{1}\right)+g_{1}\left(x_{1}\right) x_{2} \\
& \dot{x}_{2}=f_{2}\left(x_{1}, x_{2}\right)+g_{2}\left(x_{1}, x_{2}\right) x_{3} \\
& \dot{x}_{3}=f_{3}\left(x_{1}, x_{2}, x_{3}\right)+g_{3}\left(x_{1}, x_{2}, x_{3}\right) x_{4}
\end{aligned}
$$

$$
\begin{aligned}
& \dot{x}_{n-1}=f_{n-1}\left(x_{1}, x_{2}, x_{3}, \ldots, x_{n-1}\right)+ \\
& g_{n-1}\left(x_{1}, x_{2}, x_{3}, \ldots, x_{n-1}\right) x_{n} \\
& \dot{x}_{n}=f_{n}\left(x_{1}, x_{2}, x_{3}, \ldots, x_{n}\right)+g_{n}\left(x_{1}, x_{2}, x_{3}, \ldots, x_{n}\right) u
\end{aligned}
$$

where $x \in R^{n}, u \in R$. With $f_{i}(0)=0$ and $g_{i}(0) \neq 0$ for $i=1, \ldots, n . f_{i}$ and $g_{i}$ are smooth functions and are differentiable.

Stepl: Considering the first subsystem of (1), $x_{2}$ is taken as a virtual control input and choose:

$$
x_{2}=\frac{1}{g_{1}\left(x_{1}\right)}\left(u_{1}-f_{1}\left(x_{1}\right)\right)
$$

The first subsystem is changed to $\dot{x}_{1}=u_{1}$. Choosing $u_{1}=-k_{1} x_{1}$ with $k_{1}>0$, the origin of the first subsystem $x_{1}=0$ is asymptotically stable, and the corresponding Lyapunov function is $V_{1}\left(x_{1}\right)=x_{1}^{2} / 2$, then (2) is changed to:

$$
x_{2}=\phi_{1}\left(x_{1}\right)=\frac{1}{g_{1}\left(x_{1}\right)}\left(-k_{1} x_{1}-f_{1}\left(x_{1}\right)\right)
$$

Step2: Take $x_{3}$ as a virtual control input and the $\left(x_{1}, x_{2}\right)$ subsystem is changed to (5).

$$
\begin{gathered}
x_{3}=\frac{1}{g_{2}\left(x_{1}, x_{2}\right)}\left(u_{2}-f_{2}\left(x_{1}, x_{2}\right)\right) \\
\dot{x}_{1}=f_{1}\left(x_{1}\right)+g_{1}\left(x_{1}\right) x_{2} \\
\dot{x}_{2}=u_{2}
\end{gathered}
$$

which is in the form of backstepping method, so the control law $u_{2}$ is as follows:

$$
\begin{aligned}
& u_{2}=-\frac{\partial V_{1}}{\partial x_{1}} g_{1}\left(x_{1}\right)-k_{2}\left(x_{2}-\phi_{1}\left(x_{1}\right)\right)+\frac{\partial \phi_{1}}{\partial x_{1}}\left[f_{1}\left(x_{1}\right)\right. \\
& \left.+g_{1}\left(x_{1}\right) x_{2}\right]
\end{aligned}
$$

where $k_{2}>0$. This control law asymptotically stabilizes $\left(x_{1}, x_{2}\right)=(0,0)$ and Lyapunov function is as $(7)$.

$$
V_{2}\left(x_{1}, x_{2}\right)=V_{1}\left(x_{1}\right)+\frac{1}{2}\left(x_{2}-\phi_{1}\left(x_{1}\right)\right)^{2}
$$

Substituting (6) into (4) gives

$$
\begin{aligned}
& x_{3}=\phi_{2}\left(x_{1}, x_{2}\right)=\frac{1}{g_{2}\left(x_{1}, x_{2}\right)}\left[-\frac{\partial V_{1}}{\partial x_{1}} g_{1}\left(x_{1}\right)-\right. \\
& k_{2}\left(x_{2}-\phi_{1}\left(x_{1}\right)\right)+\frac{\partial \phi_{1}}{\partial x_{1}}\left(f_{1}\left(x_{1}\right)+g_{1}\left(x_{1}\right) x_{2}\right) \\
& \left.-f_{2}\left(x_{1}, x_{2}\right)\right]
\end{aligned}
$$

Step 3:

Take $x_{4}$ as a virtual control input and the $\left(x_{1}, x_{2}, x_{3}\right)$ subsystem is changed to (10).

$$
\begin{aligned}
& x_{4}=\frac{1}{g_{3}\left(x_{1}, x_{2}\right)}\left(u_{3}-f_{3}\left(x_{1}, x_{2}, x_{3}\right)\right) \\
& \dot{x}_{1}=f_{1}\left(x_{1}\right)+g_{1}\left(x_{1}\right) x_{2} \\
& \dot{x}_{2}=f_{2}\left(x_{1}, x_{2}\right)+g_{2}\left(x_{1}, x_{2}\right) x_{3} \\
& \dot{x}_{3}=u_{3}
\end{aligned}
$$

which is in the form of backstepping method, so the control law $u_{3}$ is as follows:

$$
\begin{aligned}
& u_{3}=-\frac{\partial V_{2}}{\partial x_{2}} g_{2}\left(x_{1}, x_{2}\right)-k_{3}\left(x_{3}-\phi_{2}\left(x_{1}, x_{2}\right)\right) \\
& +\frac{\partial \phi_{2}}{\partial x_{1}}\left(f_{1}\left(x_{1}\right)+g_{1}\left(x_{1}\right) x_{2}\right) \\
& +\frac{\partial \phi_{2}}{\partial x_{2}}\left(f_{2}\left(x_{1}, x_{2}\right)+g_{2}\left(x_{1}, x_{2}\right) x_{3}\right)
\end{aligned}
$$

where $k_{3}>0$. This control law asymptotically stabilizes $\left(x_{1}, x_{2}, x_{3}\right)=(0,0,0)$ and Lyapunov function is as (12).

$$
V_{3}\left(x_{1}, x_{2}, x_{3}\right)=V_{2}\left(x_{1}, x_{2}\right)+\frac{1}{2}\left(x_{3}-\phi_{2}\left(x_{1}, x_{2}\right)\right)^{2}
$$

Substituting (11) into (9) gives

$$
\begin{aligned}
& x_{4}=\phi_{3}\left(x_{1}, x_{2}, x_{3}\right)=\frac{1}{g_{3}\left(x_{1}, x_{2}, x_{3}\right)}\left[-\frac{\partial V_{2}}{\partial x_{2}} \times\right. \\
& g_{2}\left(x_{1}, x_{2}\right)-k_{3}\left(x_{3}-\phi_{2}\left(x_{1}, x_{2}\right)\right)+\frac{\partial \phi_{2}}{\partial x_{1}}\left(f_{1}\left(x_{1}\right)+\right. \\
& \left.g_{1}\left(x_{1}\right) x_{2}\right)+\frac{\partial \phi_{2}}{\partial x_{2}}\left(f_{2}\left(x_{1}, x_{2}\right)+g_{2}\left(x_{1}, x_{2}\right) x_{3}\right)- \\
& \left.f_{3}\left(x_{1}, x_{2}, x_{3}\right)\right]
\end{aligned}
$$

Step n:

Actual control law $u$ where can asymptotically stabilize (1), is as follows:

$$
\begin{aligned}
& u=\frac{1}{g_{n}\left(x_{1}, \ldots, x_{n}\right)}\left[-\frac{\partial V_{n-1}}{\partial x_{n-1}} g_{n-1}\left(x_{1}, \ldots, x_{n-1}\right)-\right. \\
& k_{n}\left(x_{n}-\phi_{n-1}\left(x_{1}, \ldots, x_{n-1}\right)\right)+\frac{\partial \phi_{n-1}}{\partial x_{1}}\left(f_{1}\left(x_{1}\right)+\right. \\
& \left.g_{1}\left(x_{1}\right) x_{2}\right)+\ldots+\frac{\partial \phi_{n-1}}{\partial x_{n-1}}\left(f_{n-1}\left(x_{1}, \ldots, x_{n-1}\right)+\right. \\
& \left.\left.g_{n-1}\left(x_{1}, \ldots, x_{n-1}\right) x_{n}\right)-f_{n}\left(x_{1}, \ldots, x_{n}\right)\right]
\end{aligned}
$$

where $k_{n}>0$. This control law asymptotically stabilizes $\left(x_{1}, \ldots, x_{n}\right)=(0, \ldots, 0)$ and Lyapunov function is as $(15)$.

$$
\begin{aligned}
& V_{n}\left(x_{1}, \ldots, x_{n}\right)=V_{n-1}\left(x_{1}, \ldots, x_{n-1}\right)+\frac{1}{2}\left(x_{n}-\right. \\
& \left.\phi_{n-1}\left(x_{1}, \ldots, x_{n-1}\right)\right)^{2}
\end{aligned}
$$




\section{OPTIMIZATIONMETHOD}

\section{A. Particle Swarm Optimization (PSO)}

In original PSO [18], each particle is analogous to an individual "fish" in a school of fish. It is a population-based optimization technique, where a population is called a swarm. A swarm consists of $\mathrm{N}$ particles moving around in a D-dimensional search space. The position of the $i$ th particle can be represented by $x_{i}=\left(x_{i 1}, x_{i 2}, \ldots, x_{i D}\right)$. The velocity for the $i$ th particle can be written as $v_{i}=\left(v_{i 1}, v_{i 2}, \ldots, v_{i D}\right)$. Each particle coexists and evolves simultaneously based on knowledge shared with neighboring particles; it makes use of its own memory and knowledge gained by the swarm as a whole to find the best solution. The best previously encountered position of the $i$ th particle is denoted its individual best position $p_{i}=\left(p_{i 1}, p_{i 2}, \ldots, p_{i D}\right)$, a value called pbest $_{i}$. The best value of the all individual pbest ${ }_{i}$ values is denoted the global best position $g_{i}=\left(g_{1}, g_{2}, \ldots, g_{D}\right)$ and called gbest. The PSO process is initialized with a population of random particles, and the algorithm then executes a search for optimal solutions by continuously updating generations. At each generation, the position and velocity of the $i$ th particle are updated by pbest ${ }_{i}$ and gbest in the swarm. The update equations can be formulated as:

$$
\begin{gathered}
v_{i d}^{\text {new }}=w \times v_{i d}^{\text {old }}+c_{1} \times r_{1} \times\left(\text { pbest }_{i d}-x_{i d}^{\text {old }}\right)+ \\
c_{2} \times r_{2} \times\left(\text { gbest }_{d}-x_{i d}^{\text {old }}\right) \\
x_{i d}^{\text {new }}=x_{i d}^{\text {old }}+v_{i d}^{\text {new }}
\end{gathered}
$$

$r_{1}$ and $r_{2}$ are random numbers between $(0,1)$, and $c_{1}$ and $c_{2}$ are acceleration constants, which control how far a particle will move in a single generation. Velocities $v_{i d}^{\text {new }}$ and $v_{i d}^{\text {old }}$ denote the velocities of the new and old particle, respectively. $x_{i d}^{\text {old }}$ is the current particle position, and $x_{i d}^{\text {new }}$ is the new, updated particle position. The inertia weight $w$ controls the impact of the previous velocity of a particle on its current one [28]. In general, the inertia weight is decreased linearly from 0.9 to 0.4 throughout the search process to effectively balance the local and global search abilities of the swarm [29]. The equation for the inertia weight $w$ can be written as:

$w=\left(w_{\max }-w_{\min }\right) \times \frac{\text { Iteration }_{\max }-\text { Iteration }_{i}}{\text { Iteration }_{\max }}+w_{\min }$

In Eq. (18), $w_{\text {max }}$ is $0.9, w_{\text {min }}$ is 0.4 and Iteration max $_{\text {ax }}$ is the maximum number of allowed iterations.

\section{B. Chaotic Particle Swarm Optimization (CPSO)}

In the field of engineering, it is well recognized that chaos theory can be applied as a very useful technique in practical application. The chaotic system can be described by a phenomenon, in which a small change in the initial condition will lead to nonlinear change in future behavior, besides that the system exhibits distinct behaviors under different phases, i.e. stable fixed points, periodic oscillations, bifurcations, and ergodicity [30]. Chaos [31] is also a common nonlinear phenomenon with much complexity and is similar to randomness. Chaos is typically highly sensitive to the initial values and thus provides great diversity based on the ergodic property of the chaos phase, which transits every state without repetition in certain ranges. It is generated through a deterministic iteration formula. Due to these characteristics, chaos theory can be applied in optimization.

In PSO, the parameters $w, r_{1}$ and $r_{2}$ are the key factors affecting the convergence behavior [32], [33]. The inertia weight controls the balance between the global exploration and the local search ability. A large inertia weight favors the global search, while a small inertia weight favors the local search. For this reason, an inertia weight that linearly decreases from 0.9 to 0.4 throughout the search process is usually used [29]. Since logistic maps are frequently used chaotic behavior maps and chaotic sequences can be quickly generated and easily stored, there is no need for storage of long sequences [34]. In CPSO, sequences generated by the logistic map substitute the random parameters $r_{1}$ and $r_{2}$ in PSO. The parameters $r_{1}$ and $r_{2}$ are modified by the logistic map based on the following equation.

$$
C r_{(t+1)}=4 \times C r_{(t)} \times\left(1-C r_{(t)}\right)
$$

In Eq. (19), $C r_{(0)}$ is generated randomly for each independent run, with $C r_{(0)}$ not being equal to $\{0,0.25,0.5,0.75,1\}$. The velocity update equation for CPSO can be formulated as:

$$
\begin{aligned}
& v_{i d}^{\text {new }}=w \times v_{i d}^{\text {old }}+c_{1} \times C r \times\left(\text { pbest }_{i d}-x_{i d}^{\text {old }}\right)+ \\
& c_{2} \times(1-C r) \times\left(\text { gbest }_{d}-x_{i d}^{\text {old }}\right)
\end{aligned}
$$

In Eq. (20), $C r$ is a function based on the results of the logistic map with values between 0.0 and 1.0. Fig. 1 shows the chaotic $\mathrm{Cr}$ value using a logistic map for 200 iterations where $C r_{(0)}=0.0001$. The pseudo-code of CPSO is shown below [21], [35].

\section{CPSO pseudo-code}

01: begin

02: Randomly initialize particles swarm

03: Randomly generate $\mathrm{Cr}_{(0)}$

04: while (number of iterations, or the stopping criterion is not met)

05: Evaluate fitness of particle swarm

06: for $\mathrm{n}=1$ to number of particles

07: $\quad$ Find pbest

08: $\quad$ Find gbest

09: $\quad$ for $\mathrm{d}=1$ to number of dimension of particle

10: $\quad$ update the Chaotic Cr value by Eq. (19)

11: update the position of particles by Eq. (20) and

Eq. (17)

12: $\quad$ next d

13: next $\mathrm{n}$

14: update the inertia weight value by Eq. (18)

15: next generation until stopping criterion

16: end 
In fact, In CPSO, a chaotic map was embedded to determine the PSO parameters $r_{1}$ and $r_{2}$. The PSO parameters $r_{1}$ and $r_{2}$ cannot ensure optimal ergodicity in the search space because they are absolutely random [36] i.e. the $r_{1}$ and $r_{2}$ are generated by a linear congruential generator (LCG) with a random seed. The generated sequence of LCG consists of pseudo-random numbers that have periodic characteristics [37]. Furthermore, the generated sequence of a logistic map also consists of pseudo-random numbers, but there are no fixed points, periodic orbits, or quasi-periodic orbits in the behavior of the chaos system [38]. As a result, the system can avoid being entrapment in local optima [20]. So we use CPSO, in this paper.

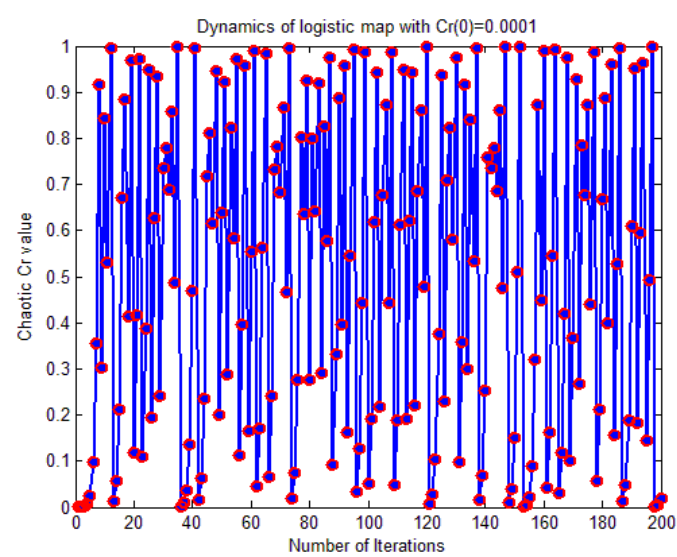

Fig. 1. Chaotic Cr value using a logistic map for 200 iterations; $\operatorname{Cr}(0)=0.0001$.

\section{Proposed InTELLIGENT BACKSTEPPING CONTROLLER}

By adopting the chaotic searching to improve the global searching performance of the particle swarm optimization (PSO), and using the improved PSO (CPSO) to optimize the key parameters of the backstepping controller, an intelligent backsteppingcontroller (IBSC) is formed. This algorithm determines the parameters of backstepping controller by minimizing the objective function. The objective function, used for controller tuning has been taken as a weighted sum of the Integral of Time multiplied Absolute Error (ITAE) and squared controller output similar to that of [39], [40], i.e.

$$
\left\{\begin{array}{l}
J=\int_{0}^{t_{f}}\left[w_{1} t|E(t)|+w_{2} u^{2}(t)\right] d t \\
|E(t)|=\sum_{i=1}^{n}\left|e_{i}(t)\right| \\
e_{i}(t)=x_{i}(t)-x_{d i}
\end{array}\right.
$$

where $t_{f}$ is the final time, in seconds. $x_{i}$ is the system state and $x_{d i}$ is the favorite mood for $x_{i}$. Based on the purpose of system for placing the state at zero value; $x_{d i}$ is equal to zero. Also, $n$ represents the system degree.

It is worth mentioning that the weights $w_{1}$ and $w_{2}$ have been introduced in the objective function (21) with a provision of balancing the impact of the error and control signal. In the present simulation study we have considered equal weights for the two objectives to be met by the controller as such the minimization of the error index is as equally important as the control signal is. The objective function $J$ in (21) is now minimized to find out the optimal set of controller parameters which simultaneously reduces the ITAE and control signal $u(t)$.In fact, in the proposed controller, the backstepping method parameters are chosen such that the time response of system states converges to zero in a short time, i.e. the system chaos is controlled faster. Besides, more limited control signal is needed for stabilization of system states and chaos control.

\section{AN ILLUSTRATED EXAMPLE}

\section{A. Genesio-Tesi Chaotic System}

The Genesio-Tesi system, proposed by Genesio and Tesi [41], is one of paradigms of chaos since it captures many features of chaotic systems. It includes a simple square part and three simple ordinary differential equations that depend on three positive real parameters. The dynamic equation of the system is as follows:

$$
\begin{aligned}
& \dot{x}_{1}=x_{2} \\
& \dot{x}_{2}=x_{3} \\
& \dot{x}_{3}=-a x_{3}-b x_{2}-c x_{1}+x_{1}^{2}
\end{aligned}
$$

where $x_{i}, i=1,2,3$ are state variables, and $a, b$ and $c$ are the positive real constants satisfying $a b<c$. For instance, the system is chaotic for the parameters $a=1.2, b=2.92$, $c=6$. For the initial condition $\left(x_{1}, x_{2}, x_{3}\right)=(0.1,-0.2,0.2)$ , the chaotic motion of the system is illustrated in Figs. 2 and 3.

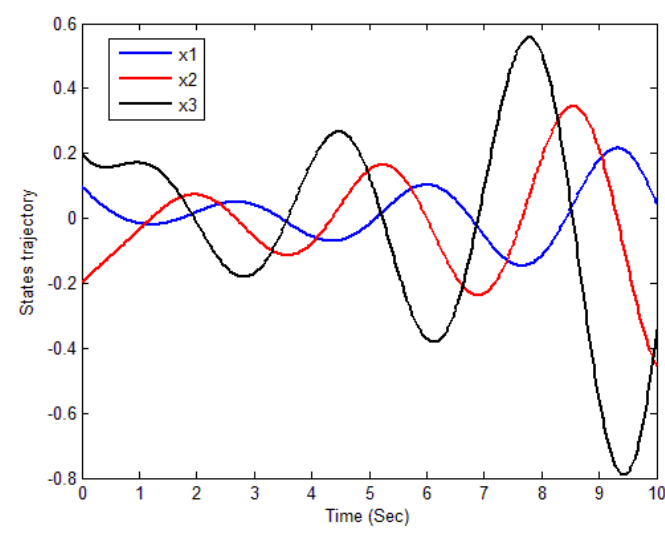

Fig. 2. Chaotic motion of states.

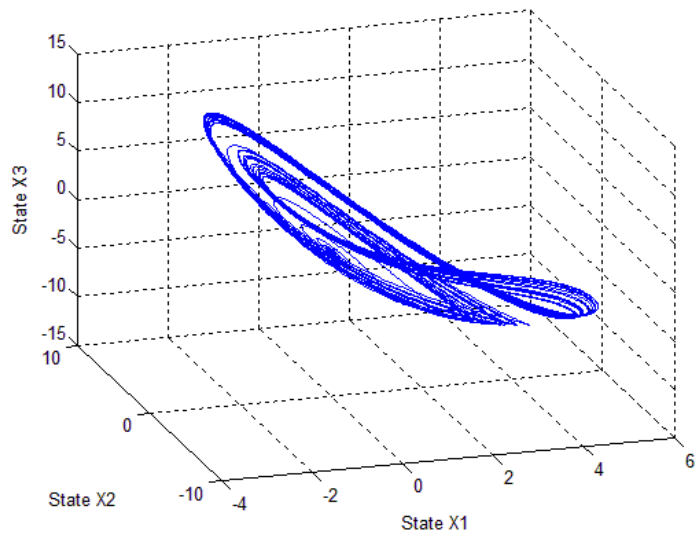

Fig. 3. Chaotic attractor (0-250sec). 


\section{B. Controlling Genesio-Tesi Chaotic System}

As shown in Figs. 2 and 3, the system has chaotic behavior when the control input does not apply.In this section, the backstepping method is utilized for the control of chaos of the Genesio-Tesi system. For this purpose, a control signal $u$ is added to the equation (1). The system (1) is rewritten, as following:

$$
\begin{aligned}
& \dot{x}_{1}=x_{2} \\
& \dot{x}_{2}=x_{3} \\
& \dot{x}_{3}=-a x_{3}-b x_{2}-c x_{1}+x_{1}^{2}+u
\end{aligned}
$$

Backstepping method is used to set states $x_{1}, x_{2}, x_{3}$ to the origin point $(0,0,0)$ via the control signal $u$ calculated with threesteps.According to Section II, the design procedure is as follows:

Step 1: $x_{2}$ is taken as (26) to construct the Lyapunovfunction (25) for (24).

$$
\begin{gathered}
\dot{x}_{1}=x_{2} \\
V_{1}\left(x_{1}\right)=x_{1}^{2} / 2 \\
x_{2}=\phi_{1}\left(x_{1}\right)=-k_{1} x_{1}
\end{gathered}
$$

Step2: Take virtual control input (28) and Lyapunov function (29) for $\left(x_{1}, x_{2}\right)$ of (27).

$$
\begin{gathered}
\dot{x}_{1}=x_{2} \\
\dot{x}_{2}=x_{3} \\
x_{3}=\phi_{2}\left(x_{1}, x_{2}\right)=-\left(1+k_{1} k_{2}\right) x_{1}-\left(k_{1}+k_{2}\right) x_{2} \\
V_{2}\left(x_{1}, x_{2}\right)=\frac{1}{2} x_{1}^{2}+\frac{1}{2}\left(x_{2}+k_{1} x_{1}\right)^{2}
\end{gathered}
$$

Step 3: Final control input and Lyapunov function are given in (31) and (32) for (30).

$$
\begin{gathered}
\dot{x}_{1}=x_{2} \\
\dot{x}_{2}=x_{3} \\
\dot{x}_{3}=-a x_{3}-b x_{2}-c x_{1}+x_{1}^{2}+u \\
u=a x_{3}+b x_{2}+c x_{1}-x_{1}^{2}-\left(k_{1}+k_{3}+k_{1} k_{2} k_{3}\right) x_{1}- \\
\left(2+k_{1} k_{2}+k_{1} k_{3}+k_{2} k_{3}\right) x_{2}-\left(k_{1}+k_{2}+k_{3}\right) x_{3} \\
V_{3}\left(x_{1}, x_{2}, x_{3}\right)=\frac{1}{2} x_{1}^{2}+\frac{1}{2}\left(x_{2}+k_{1} x_{1}\right)^{2}+\frac{1}{2}\left[x_{3}+\right. \\
\left.\left(1+k_{1} k_{2}\right) x_{1}+\left(k_{1}+k_{2}\right) x_{2}\right]^{2}
\end{gathered}
$$

According to Eq. (31), it is observed that the control signal consists of the parameters which are positive. These parameters have to be chosen properly. Improper choice of the parameters causes improper performance and even instability of the system. Besides, finding the parameters through trial and error is so time-consuming. The CPSO algorithm obtains the proper values of the parameters via minimizing the fitness function. The parameters of the CPSO algorithm are set as shown in Table I. The sampling time in this simulation is 0.02 . In the proposed controller, the searching ranges for the backstepping parameters $k_{1}, k_{2}$, and $k_{3}$ are limited to $[0,10]$. The backstepping parametersare obtained for 20 iterations. In this example $t_{f}$ is equal to 10 seconds.Besides, the weights $w_{1}$ and $w_{2}$ of fitness function are chosen as 0.5. $n$ represents the system degree and is equal to 3 in this example.

TABLE I: PARAMETERS USED IN THE CPSO

\begin{tabular}{lc}
\hline \hline Population size & 20 \\
Acceleration constant $c_{1}$ & 2 \\
Acceleration constant $c_{2}$ & 2 \\
Inertia weight $w$ & started from 0.9 and decreased linearly \\
Number of iterations & to 0.4 \\
\hline \hline
\end{tabular}

The parameters of backstepping controller are obtained by using CPSO algorithm, as follows: $k_{1}=4.5908, k_{2}=3.9283$, $k_{3}=4.5908$. The search process of CPSO algorithm for finding the parameters is shown in Fig. 4. Besides, the fitness value obtained by the algorithm is 0.1084 . The trajectory of fitness variations with respect to algorithm iteration is shown in Fig.5.

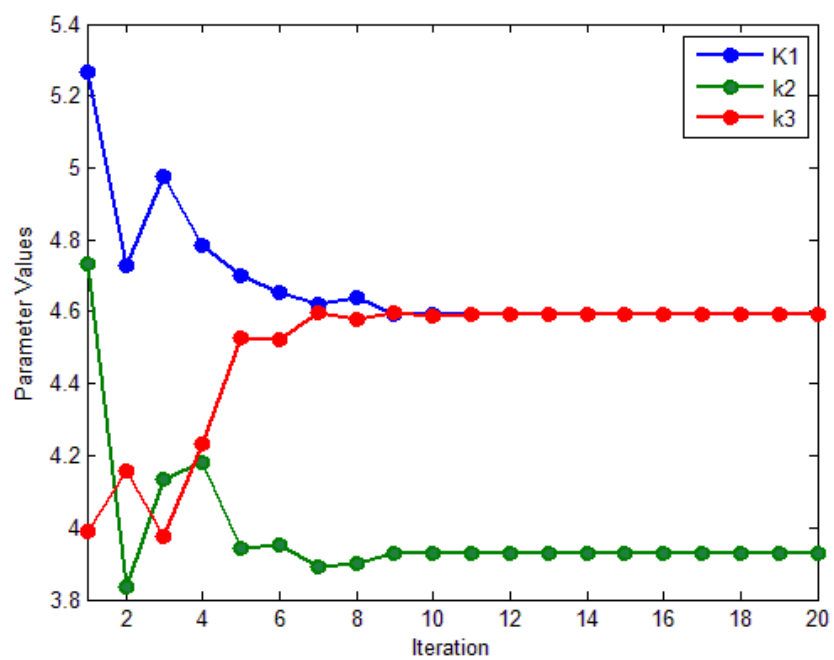

Fig. 4. The parameter values trajectory.

The parameter setting of Table Iis based on the experiments accomplished by Kennedy, Eberhart and Shi. The two factors $c_{1}$ and $c_{2}$ are learning factors, $c_{1}=c_{2}=2$ [42].Also, the inertia weight $w$ is critical for the convergence behavior of PSO. A suitable value for the inertia weight $\omega$ usually provides a balance between global and local exploration abilities and consequently results in a better optimum solution. Initially, the inertia weight was constant. However, experimental results indicated that it is better to initially set the inertia to a large value in order to promote global exploration of the search space and decrease it to get more refined solutions. Therefore, a decaying inertia weight starting at 0.9 and ending at 0.4 was used for PSO [43].

Therefore, by choosing $c_{1}=c_{2}=2$ and decreasing inertia weight linearly from 0.9 to 0.4 , the CPSO algorithm shows the best performance. Based on this result, the mentioned values are chosen for the parameters.

Also, the population size must be chosen properly. If small 
population size is chosen, the algorithm search ability will decrease and the algorithm will not have a proper performance. On the other part, the choice of big population size leads to good algorithm search and the convergence rate increases, but the algorithm run time will be high.

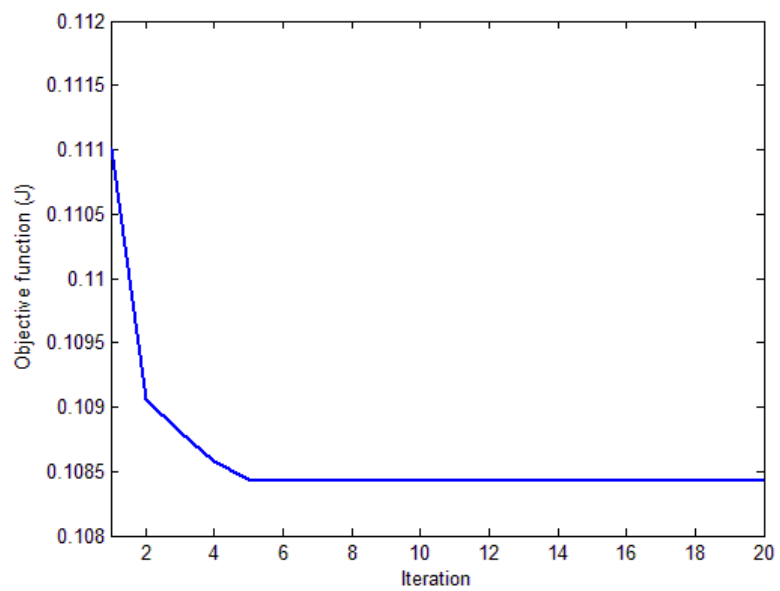

Fig. 5. The Objective function trajectory.

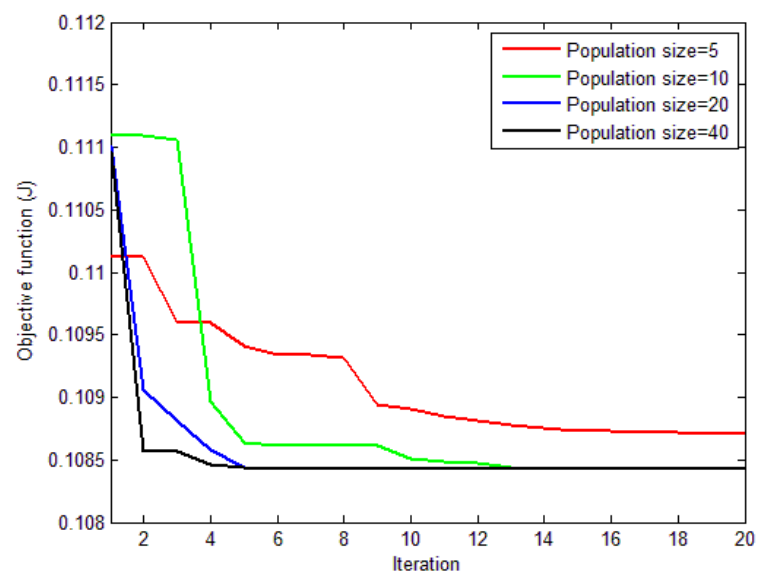

Fig. 6. Comparison of convergence ofobjective functionfor different population sizes.

Fig. 6 shows the fitness variations curve with respect to algorithm iterations for different population sizes.Meanwhile, the diagrams of the variations of backstepping method parameters with respect to algorithm iterations are shown in Figs. 7, 8 and 9 for different population sizes.According to Figs. 6-9, if the population size is chosen as 5, 10, 20 and 40 in order to optimize the backstepping method, it is observed that the small population size (i.e. 5) does not have a suitable performance. In addition, the population size10 needs more iterations to reach the optimal point and also, does not have an adequate convergence rate. In spite of the fact that big population size, for example 40, decreases the required iterations to reach the optimal point, but the algorithm run time is very high. Therefore, in order to reach the optimal point in fewer iterations and also, to have loweralgorithm run time, the population size 20 is utilized in this work, which leads to acceptable results.

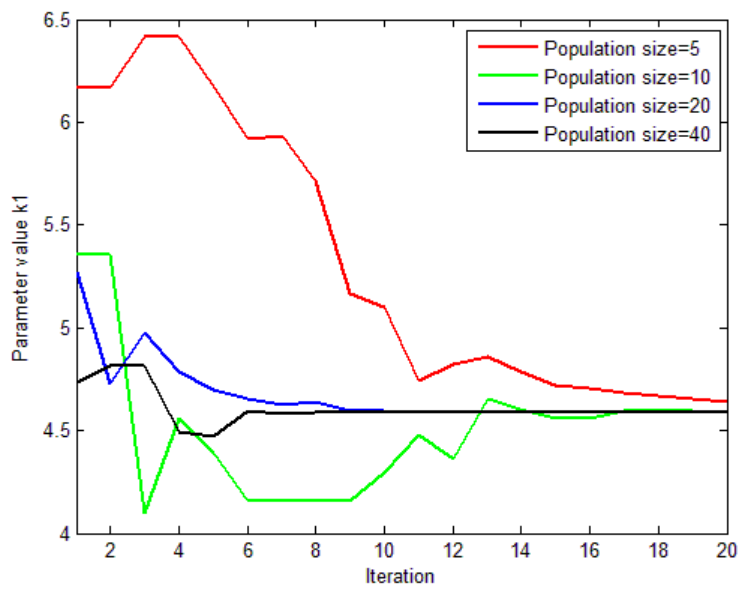

Fig. 7. Comparison of trajectories of parameter $\mathrm{k} 1$ for different population sizes.

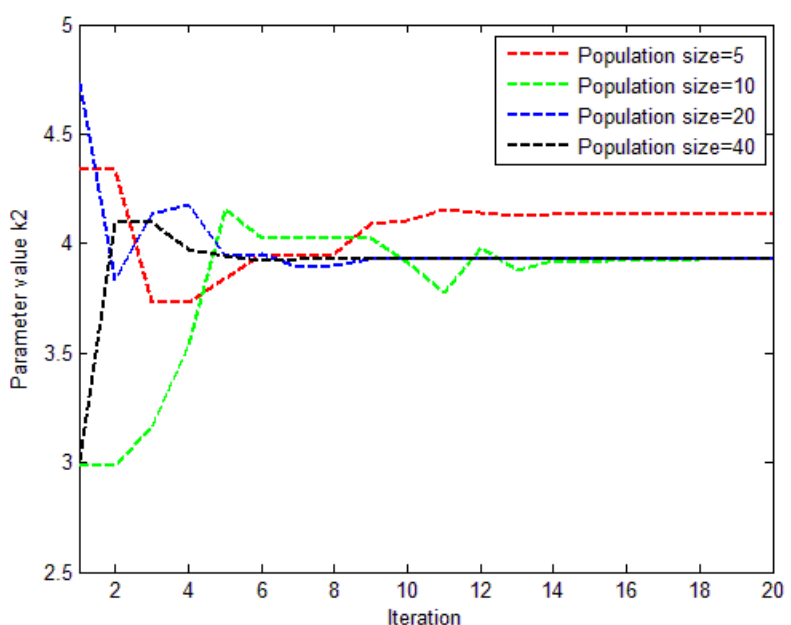

Fig. 8. Comparison of trajectories of parameter $\mathrm{k} 2$ for different population sizes.

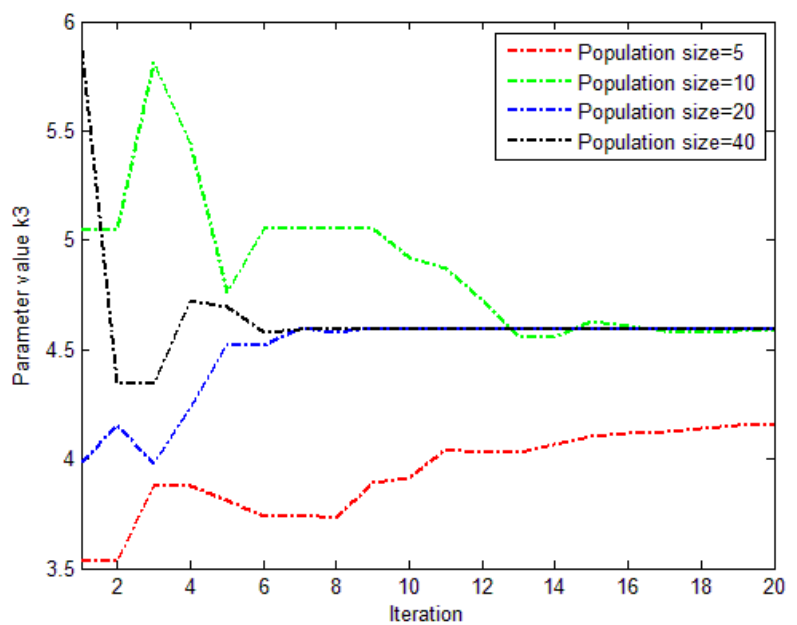

Fig. 9. Comparison of trajectories of parameter $\mathrm{k} 3$ for different population sizes.

The time response of the states of Genesio system after applying the controller is shown in Figs. 10-12. The controlled chaos of the system is demonstrated in Fig. 13. Also, the control signal is illustrated in Fig. 14. 


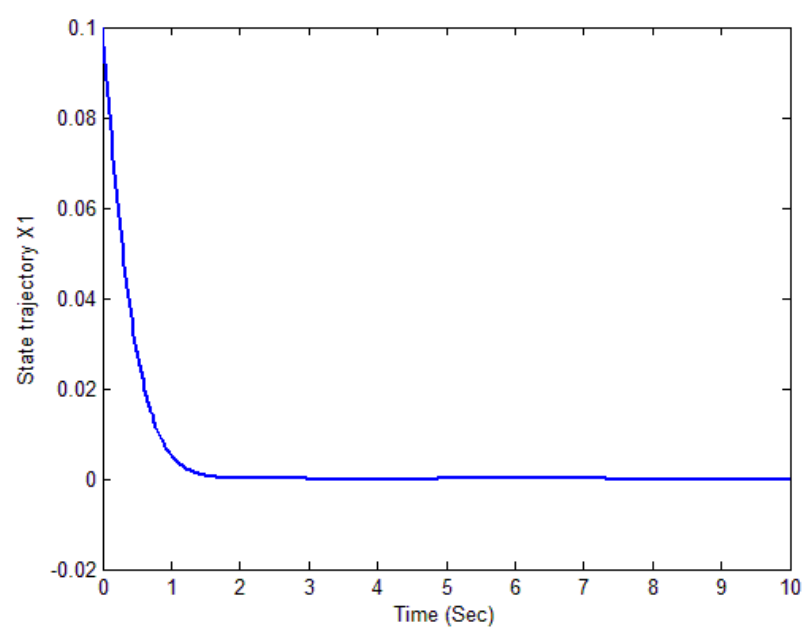

Fig. 10. Controlled time response of the state $\mathrm{X}_{1}$.

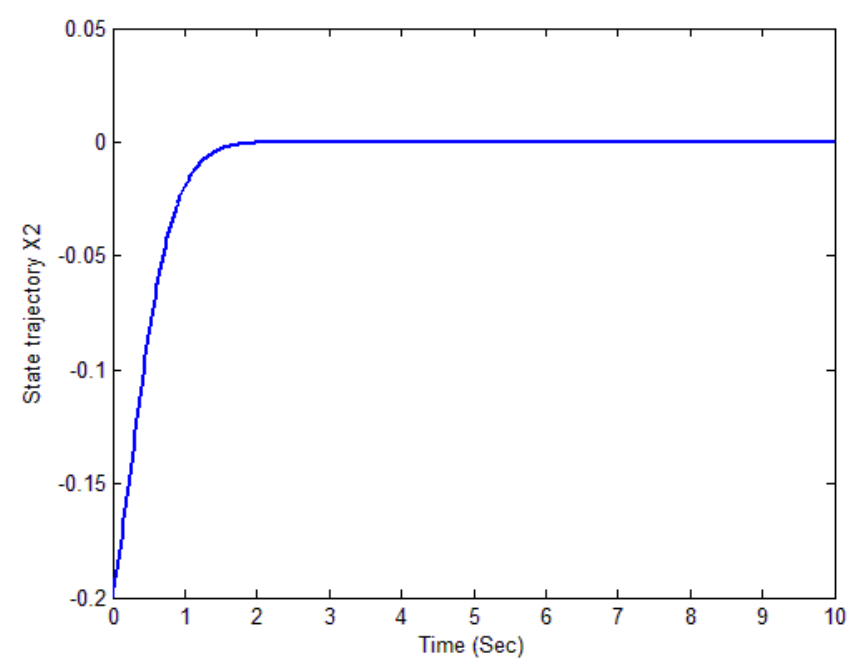

Fig. 11. Controlled time response of the state $\mathrm{x}_{2}$.

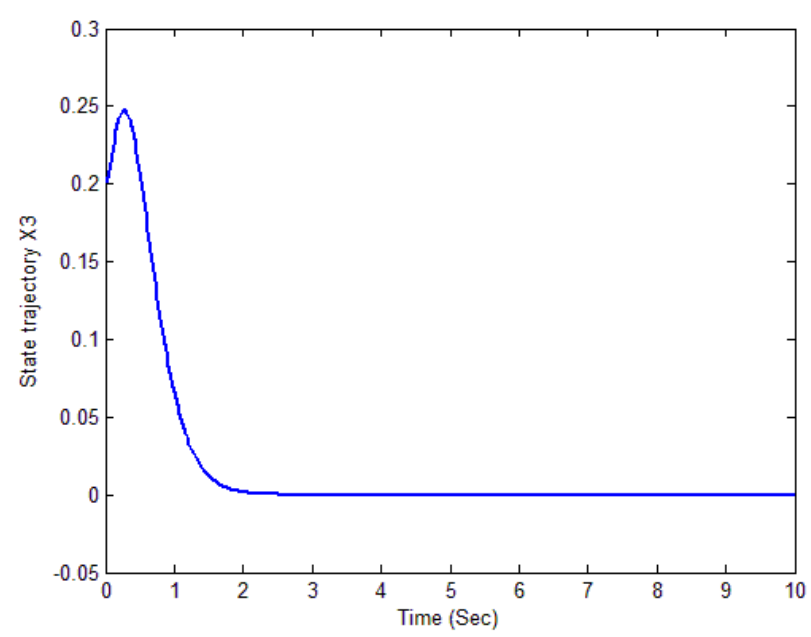

Fig. 12. Controlled time response of the state $\mathrm{x}_{3}$.

As shown in Figs.10-13, the CPSO algorithm causes the states of the system converge to zero in a shorter time by minimizing the fitness function and in consequence, the system chaos is controlled in much shorter time. In addition, according to Fig. 14, it is observed that the proposed controller has created a limited control signal to chaos control of Genesio system. Because the control effort is applied, in the proposed objective function.Then, by minimizing the objective function, the saturation of control signal is avoided.

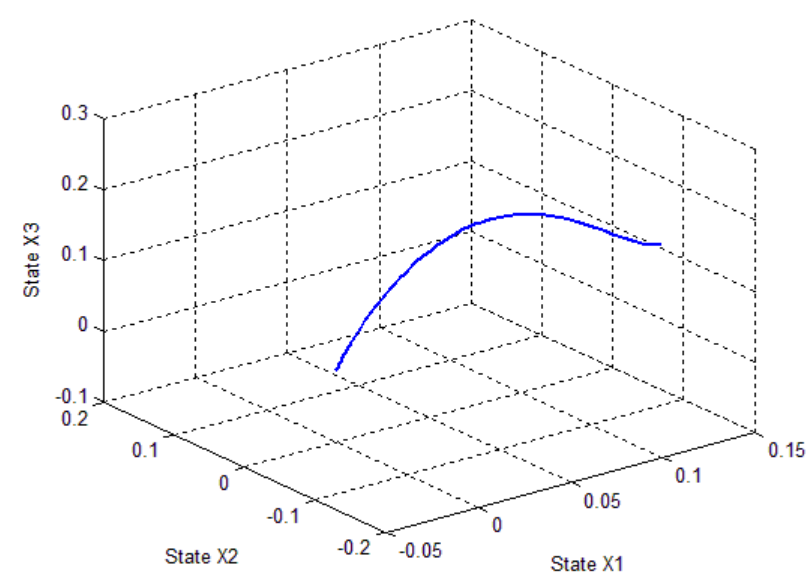

Fig. 13. Controlled Chaotic attractor $(0-250 \mathrm{sec})$.

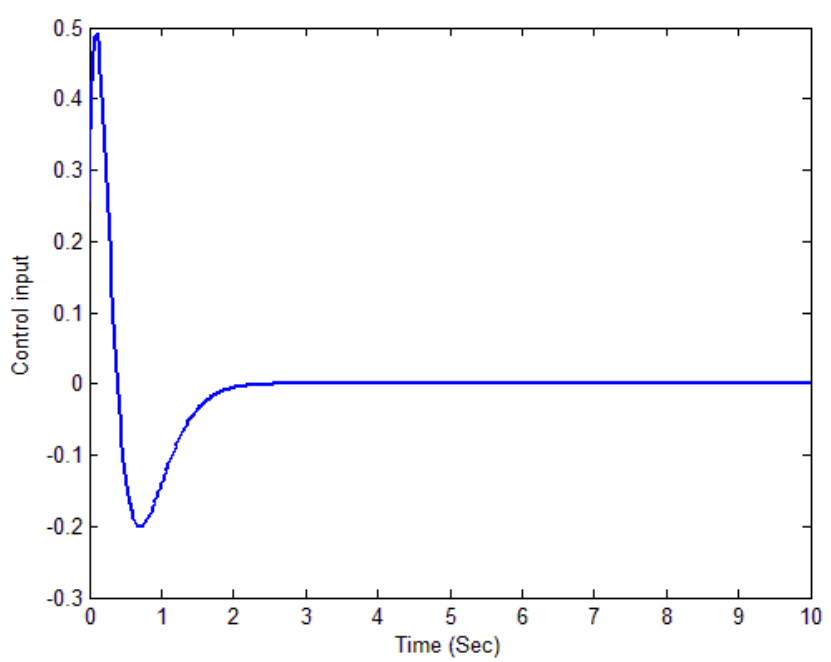

Fig. 14. The control law u.

\section{CONCLUSION}

In this paper, a new nonlinear controller named "intelligent backstepping controller" is established by combination of backstepping method and CPSO algorithm. This new controller is utilized for chaos control of Genesio-Tesi system.The CPSO algorithm determines the optimal parameters of the backstepping method by minimizing the objective function. By this new controller, the fastest response is achieved with the least control effort. The simulation results of Genesio-Tesi chaotic system show the effectiveness of the proposed controller and the tuning process.

\section{REFERENCES}

[1] E. Ott, C. Grebogi, and J. A. Yorke, "Controlling chaos," Phys Rev Lett,vol. 64, pp. 1196-9,1990.

[2] T.L. Vincent, and J. Yu, "Control of a chaotic system," Dynam Control, vol. 1, pp. 35-52, 1991.

[3] R. Luce, and J. P. Kernevez, "Controllability of Lorenz equation," Int Series Nume Math, vol. 97, pp. 257-261,1991.

[4] T. H. Yeap and N. U. Ahmed, "Feedback control of chaotic systems,"Dynam Control, vol. 4, pp. 97-114, 1994.

[5] J. A. Gallegos, "Nonlinear regulation of a Lorenz system by feedback linearization techniques," Dynam Control, vol.4, pp. 277-98, 1994.

[6] C. C. Fuh, P. C. Tung, "Controlling Chaos using differential geometric method," Phys Rev Lett, vol.75, pp. 2952-5, 1995.

[7] Y. Zeng, and S. N. Singh, "Adaptive control of chaos in Lorenz system,” Dynam Control, vol. 7, pp. 143-54, 1997. 
[8] M. D. Bernardo, "An adaptive approach to the control and synchronization of continuous time chaotic systems,"Int. J. Bifurcation Chaos, vol. 6, pp. 557-68, 1996.

[9] M. Feki, "An adaptive feedback control of linearizable chaotic systems," Chaos, Solitons\& Fractals, vol. 15, pp. 883-90, 2003.

[10] C. W. Wu, T. Yang, and L. O. Chua, "On adaptive synchronization and control of nonlinear dynamical systems,"Int. J. Bifurcation Chaos, vol. 6, pp. 455-71, 1996.

[11] J. A. K. Suykens, P. F. Curran, and J. Vandewalle, "Robust Nonlinear H1synchronization of chaotic Lur'e systems,"IEEE Trans. Circuits Syst I, vol. 44, no. 10, pp. 891-904, 1997.

[12] G. Chen, X. Dong, "From chaos to order: methodologies, perspectives and applications,"World Scientific World Scientific Publishing Company, 1998.

[13] J. J. Yan , "Design of robust controllers for uncertain chaotic systems with nonlinear inputs," Chaos, Solitons \& Fractals, vol. 19, pp. 541-7, 2004.

[14] J. Lu, and S. Zhang , "Controlling Chen's chaotic attractor using backstepping design based on parameters identification," PhysLett A, vol. 286, pp. 145-9, 2001.

[15] J. H. Park, "Synchronization of Genesio chaotic system via backstepping approach," Chaos, Solitons\& Fractals, vol. 27, pp 1369-75.2006

[16] S. Bowong, F. M. Moukam Kakmeni, "Chaos control of uncertain chaotic systems via backstepping approach," ASME J. VibratAcoust, vol. 128, pp. 21-7,2006.

[17] M. T. Yassen, "Chaos control of chaotic dynamical systems using backstepping design," Chaos, Solitons \& Fractals, vol. 27, pp. 537-48, 2006.

[18] J. Kennedy, and R.C. Eberhart, "Particle swarm optimization," in: Proc. IEEE International Conference on Neural Networks 4, Perth, Australia,pp. 1942-1948, 1995.

[19] P. J. Angeline, "Evolutionary optimization versus particle swarm optimization: philosophy and performance differences,"Evolutionary programming, vol. VII, Springer, pp. 601-10, 1998

[20] B. Liu, L. Wang, Y.H. Jin, F. Tang, and D.X. Huang, "Improved particle swarm optimization combined with chaos," Chaos, Solitons and Fractals, vol. 25,pp. 1261- 1271, 2005.

[21] L.-Y. Chuang, C.-J. Hsiao, and C.-H. Yang, "Chaotic particle swarm optimization for data clustering,"Expert systems with Applications, vol. 38, pp. 14555-14563, 2011.

[22] W.-C. Hong, "Chaotic particle swarm optimization algorithm in a support vector regression electric load forecasting model,"Energy Conversion and Management, vol. 50, pp. 105-117, 2009.

[23] X. Tang, L. Zhuang, J. Cai, and C. Li, "Multi-fault classification based on support vector machine trained by chaos particle swarm optimization,"Knowledge-Based Systems, vol. 23, pp. 486-490, 2010.

[24] P. Acharjee, and S.K. Goswami, "Chaotic particle swarm optimization based robust load flow,"Electrical Power and Energy Systems, vol. 32, pp. 141-146, 2010

[25] J. Wang, S. Zhu, W. Zhao, and W. Zhu, "Optimal parameters estimation and input subset for grey model based on chaotic particle swarm optimization algorithm,"Expert Systems with Applications, vol. 38, pp. 8151-8158, 2011.

[26] Leandro dos Santos Coelho, and Antonio Augusto Rodrigues Coelho, "Model-free adaptive control optimization using a chaotic particle swarm approach," Chaos, Solitons and Fractals, vol. 41, pp 2001-2009, 2009.

[27] Y. Wang, and J.H. Liu, "Chaotic particle swarm optimization for assembly sequence planning,"Robotics and Computer-Integrated Manufacturing, vol.26, pp. 212-222, 2010.

[28] Y. Shi, and R.C. Eberhart, "A modified particle swarm optimizer,"in: Proc. IEEE International Conference on Evolutionary Computation, Anchorage, AK, 2002, pp. 69-73.

[29] Y. Shi, and R.C. Eberhart, "Empirical study of particle swarm optimization,"in: Proc. Congress on Evolutionary Computation, Washington, DC, 2002, pp. 1945-1949.

[30] L. dos Santos Coelho, andB. M. Herrera, "Fuzzy identification based on a chaotic particle swarm optimization approach applied to a nonlinear yo-yo motion system,"IEEE Transactions on Industrial Electronics, vol. 54, pp. 3234-3245, 2007.

[31] H. Lu, H. M. Zhang, and L. H. Ma, "A new optimization algorithm based on chaos,"Journal of Zhejiang University Science A, vol. 7, pp. 539-542, 2006.

[32] I. C. Trelea, "The particle swarm optimization algorithm: convergence analysis and parameter selection,"Information Processing Letters, vol.85,pp. 317-325, 2003.

[33] S. Naka, T. Genji, T. Yura, and Y. Fukuyama, "A hybrid particle swarm optimization for distribution state estimation,"IEEE Transactions on Power Systems, vol. 18,pp. 60-68, 2003.

[34] H. Gao, Y. Zhang, S. Liang, and D. Li, "A new chaotic algorithm for image encryption,"Chaos, Solitons and Fractals, vol.29, pp. 393-399, 2006.

[35] L. Y. Chuang, S.-W. Tsai, and C.-H. Yang, "Chaotic catfish particle swarm optimization for solving global numerical optimization problems,"Applied mathematics and computation,vol. 217,pp 6900-6916, 2011.

[36] J. Chuanwen, and E. Bompard, "A self-adaptive chaotic particle swarm algorithm for short term hydroelectric system scheduling in deregulated environment,"Energy Conversion and Management,vol. 46,pp. 2689-2696, 2005

[37] D. E. Knuth, "The Art of Computer Programming, Seminumerical Algorithms," Third ed., vol. 2, Addison-Wesley, 1997, pp. 10-26. Section 3.2.1: The Linear Congruential Method.

[38] D. Kuo, "Chaos and its computing paradigm,"IEEE Potentials Magazine, vol. 24,pp. 13-15, 2005.

[39] J.-Y. Cao, and B.-G. Cao, "Design of fractional order controllers based on particle swarm optimization," inProc. 1st IEEE industrial electronics and applications, 2006, pp. 1-6.

[40] J.-Y. Cao, J. Liang, and B.-G. Cao, "Optimization of fractional order PID controllers based on Genetic Algorithms," in: Proc. the 2005 international conference on machine learning and cybernetics. vol. 9. pp. 5686-9.

[41] R. Genesio, and A. Tesi, "A harmonic balance methods for the analysis of chaotic dynamics in nonlinear systems," Automatica,vol. 28,pp. 531-548,1992.

[42] J. Kennedy, R. C. Eberhart, and Y. Shi, “Swarm Intelligence,"Morgan Kaufman, San Mateo, CA, 2001.

[43] R. C. Eberhart, andY.Shi, "Particle swarm optimization: developments, applications and resources," in Proc. IEEE International Conference on Evolutionary Computation,2001, pp. 81-86. 